\title{
Linking density functional and density-matrix theory: Picosecond electron relaxation at the $\mathrm{Si}(100)$ surface
}

\author{
N. Buecking, ${ }^{1,2}$ P. Kratzer, ${ }^{2,3}$ M. Scheffler, ${ }^{2}$ and A. Knorr ${ }^{1, *}$ \\ ${ }^{1}$ AG Nichtlineare Optik und Quantenelektronik, Institut für Theoretische Physik, Technische Universität Berlin, \\ Hardenbergstrasse 36 PN 7-1, 10623 Berlin, Germany \\ ${ }^{2}$ Fritz-Haber-Institut der Max-Planck-Gesellschaft, Faradayweg 4-6, 14195 Berlin, Germany \\ ${ }^{3}$ Fachbereich Physik, Universität Duisburg-Essen, Lotharstrasse 1, 47048 Duisburg, Germany
}

(Received 20 March 2008; published 11 June 2008)

\begin{abstract}
We describe an approach that links the density-matrix theory for electron transport and relaxation with the density-functional theory for electronic structure. Our analysis of the electron dynamics at $\mathrm{Si}(100)$ reveals an unanticipated phonon bottleneck between bulklike and surface states. The fastest relaxation process observed in recent two-photon photoemission experiments is in good agreement with the calculated phonon-mediated intrasurface-band scattering.
\end{abstract}

DOI: 10.1103/PhysRevB.77.233305

PACS number(s): 73.20. $-\mathrm{r}, 78.47 .-\mathrm{p}, 71.15 .-\mathrm{m}$

Nanometer-scale semiconductor devices are increasingly affected by the electronic and phononic properties of surfaces and interfaces. In particular, the dynamics of charge carriers has become an important field of research. ${ }^{1-3}$ For example, time-resolved two-photon photoemission ${ }^{1,4-6}$ (2PPE) probes the time- and energy-resolved electron dynamics. An intriguing challenge is to understand the electron transfer dynamics between surface and bulklike states. ${ }^{5-7}$

In this Brief Report, we present a theory for the optical excitation and subsequent phonon-assisted relaxation ${ }^{8}$ of a nonequilibrium electron distribution. Specifically, we link the density-functional theory (DFT) with the density-matrix theory (DMT). ${ }^{9}$ The strength of DMT-based approaches lies in their direct treatment of the time domain, which provides a transparent description of both the electron dephasing and the relaxation processes over a wide range of time scales, while requiring just a few empirical materials parameters. Such methods have been used with great success for describing carrier relaxation after optical excitation in bulk or embedded nanostructured semiconductors. ${ }^{10-16}$ For surfaces, however, a reliable description must include more materialspecific details than typically known experimentally. The approach presented here is as follows: First, kinetic equations are derived from the DMT of open systems. Second, the parameters entering these equations are calculated by using the DFT. To demonstrate the method and motivated by recent 2PPE experiments, in this Brief Report, we address the dynamics of electron-phonon scattering ${ }^{17}$ at the $\mathrm{Si}(100)$ surface. For this problem, as for many other applications with a small electronic excitation density, the electronic and phononic states independently calculated for the ground state structure form a suitable basis in the many-particle Fock space (for substantial structural changes, cf. Ref. 18). By using the operators $a_{n \mathbf{k}}^{\dagger}, a_{n \mathbf{k}}, b_{i \mathbf{q}}^{\dagger}$, and $b_{i \mathbf{q}}$, which are the creation and annihilation operators for an electron $(a)$ and phonon $(b)$ with quantum numbers $n$ and $\mathbf{k}$ [band index and two-dimensional (2D) wave vector] and $i$ and $\mathbf{q}$ (mode index and $2 \mathrm{D}$ wave vector), respectively, the relevant Hamiltonian in second quantization reads

$$
\begin{aligned}
H= & \sum_{n \mathbf{k}} \epsilon_{n \mathbf{k}} a_{n \mathbf{k}}^{\dagger} a_{n \mathbf{k}}+\sum_{i \mathbf{q}} \hbar \omega_{i \mathbf{q}} b_{i \mathbf{q}}^{\dagger} b_{i \mathbf{q}}+\sum_{n \mathbf{k}, n^{\prime} \mathbf{k}^{\prime}} \mathbf{A}(t) \mathbf{p}_{n \mathbf{k}}^{n^{\prime} \mathbf{k}^{\prime}} a_{n \mathbf{k}}^{\dagger} a_{n^{\prime} \mathbf{k}^{\prime}} \\
& +\sum_{n \mathbf{k}, n^{\prime} \mathbf{k}^{\prime}, i \mathbf{q}} D_{n \mathbf{k}, n^{\prime} \mathbf{k}^{\prime}, i \mathbf{q}} a_{n \mathbf{k}}^{\dagger} a_{n^{\prime} \mathbf{k}^{\prime}}\left(b_{i-q}^{\dagger}+b_{i \mathbf{q}}\right) .
\end{aligned}
$$

The quantities $\epsilon_{n \mathbf{k}}$ are the band energies of single-particle excitations, which are obtained by applying an appropriate self-energy correction $\left\langle\varphi_{n \mathbf{k}}\left|\Sigma-V_{x c}\right| \varphi_{n \mathbf{k}}\right\rangle$ to the Kohn-Sham band energies $\epsilon_{n \mathbf{k}}^{K S} \cdot{ }^{19}$ We refer to the states labeled $\varphi_{n \mathbf{k}}$ as quasiparticles because the correction procedure described already accounts for most of the electron-electron interactions. The Hamiltonian incorporates the coupling of the electrons to the optical field as well as electron-phonon coupling: ${ }^{17}$ The vector potential $\mathbf{A}(t)$ in radiation gauge couples to the electrons via the momentum matrix element $\mathbf{p}_{n \mathbf{k}, n^{\prime} \mathbf{k}^{\prime}}$. This description is general if we think of $\mathbf{E}(t)=-\partial \mathbf{A}(t) / \partial t$ as an effective electric field that incorporates local field effects due to the dielectric polarization of the sample. Also, light propagation effects can in principle be treated ${ }^{20}$ but are unimportant for the present application. The phonon spectrum is $\omega_{i q}$, and $D_{n \mathbf{k}, n^{\prime} \mathbf{k}^{\prime}, i \mathbf{q}}$ are the electron-phonon coupling matrix elements. For optical absorption spectra, the electron-hole interaction was found to be quite important. ${ }^{21}$ Our present approach could be extended to include the Coulomb interaction (besides the dressing that is already included in the description of the initial states), by adding a two-particle interaction in the Hamiltonian. In this Brief Report, the use of independent quasiparticles as a starting point in Eq. (1) appears justified by previous findings ${ }^{22}$ that electron-phonon interaction dominates over Coulomb scattering for not too large quasiparticle population. Moreover, the present approach ignores exciton formation, but according to experiment, ${ }^{6}$ this is expected to play a role only on much longer time scales than those considered. The time dependence of the populations $f_{n \mathbf{k}}=\left\langle a_{n \mathbf{k}}^{\dagger} a_{n \mathbf{k}}\right\rangle$ is determined by the equations of motion of the operators $a_{n \mathbf{k}}^{\dagger}$ and $a_{n \mathbf{k}}$. The hierarchy of equations for these operators is transformed into a closed system by using the second-order Born approximation. This is a well-established approximation for materials with weak polaronic effects, ${ }^{23}$ such as bulk Si. Space charge effects are accessible to our 
method $^{24}$ but are presently neglected. Since the phononassisted relaxation of hot electrons is slow compared to the oscillation period of the phonons, ${ }^{25}$ we additionally use the Markovian approximation (energy conservation in each individual process). Due to the low population of excited electrons, their overall effect on the phonon system is small. Therefore, all phonon modes are assumed to be in thermal equilibrium at temperature $T .7,10,15$ The resulting dynamics can be cast into the form of a master equation, ${ }^{17}$

$$
\frac{d}{d t} f_{n \mathbf{k}}=\Gamma_{n \mathbf{k}}^{\text {in }}\left(1-f_{n \mathbf{k}}\right)-\Gamma_{n \mathbf{k}}^{\text {out }} f_{n \mathbf{k}} .
$$

Deriving this equation from DMT provides us with microscopic expressions for the scattering matrices $\Gamma_{n \mathbf{k}}^{\text {in }}$ and $\Gamma_{n \mathbf{k}}^{\text {out }}$. They depend both on the temperature $T$ (through the phonon occupation numbers) and on the electronic occupation densities $f_{n \mathbf{k}}$. Similar scattering contributions are valid for the microscopic polarizations $\left\langle a_{n \mathbf{k}}^{\dagger} a_{n^{\prime} \mathbf{k}^{\prime}}\right\rangle .^{10}$

While a full solution of Eq. (2) is feasible also including optical excitation, we use a two-step approach for conceptual clarity: First, initial conditions for the $f_{n \mathbf{k}}$ in Eq. (2) are derived from the optical pulse alone. The dynamical relaxation of these initial occupations $f_{n \mathbf{k}}$ that is due to electron-phonon scattering is then calculated by numerically solving Eq. (2) without an electrical field. This approach is justified by the fact that the optical pulses used in 2PPE experiments ${ }^{5,6,26}$ are typically short $\left(t_{\text {pulse }}<100 \mathrm{fs}\right)$ compared to the time scale of phonon-assisted relaxation. By separating the two regimes of excitation and phononic relaxation, the initial occupations $f_{n \mathbf{k}}$ can be analytically obtained by integrating the electronfield interaction. ${ }^{27}$ The matrix elements $\mathbf{p}_{n \mathbf{k}, n^{\prime} \mathbf{k}^{\prime}}$ and $D_{n \mathbf{k}, n^{\prime} \mathbf{k}^{\prime}, i \mathbf{q}}$, the quasiparticle band structure $\epsilon_{n \mathbf{k}}$, and the phonon dispersion $\omega_{i \mathbf{q}}$ are material specific. These quantities occurring in $\Gamma_{n \mathbf{k}}^{\text {in }}$ out are time-independent input parameters to the dynamical equations, and they should be computed for each system by first-principles calculations.

In order to demonstrate these concepts, we apply them to the $(2 \times 1)$ reconstructed $\mathrm{Si}(100)$ surface. The self-energy correction $\left\langle\varphi_{n \mathbf{k}}\left|\Sigma-V_{x c}\right| \varphi_{n \mathbf{k}}\right\rangle$ evaluated in the $G_{0} W_{0}$ approximation is practically independent of the wave vector $\mathbf{k}$ (see, e.g., Refs. 28 and 29). Thus, the quasiparticle energies of the unoccupied states are given by the Kohn-Sham levels simply shifted upward by $0.62 \mathrm{eV}$, while unchanged Kohn-Sham wave functions $\varphi_{n \mathbf{k}}$ are used to calculate the matrix elements $\mathbf{p}_{n \mathbf{k}, n^{\prime} \mathbf{k}^{\prime}}$ and $D_{n \mathbf{k}, n^{\prime} \mathbf{k}^{\prime}, i \mathbf{q}}$

The $(2 \times 1)$ reconstructed $\mathrm{Si}(100)$ surface with buckled $\mathrm{Si}$ dimers $^{28,30}$ is modeled by a supercell (repeated slab) approach by using $30 \mathrm{Si}$ layers with the bottom layer terminated by hydrogen atoms. Our tests showed that such a high number of layers is indeed necessary to ensure converged results. The electronic ground state and the Kohn-Sham wave functions are calculated by using a plane wave basis set together with $a b$ initio norm-conserving pseudopotentials, a cutoff energy of 10 Ry and a bulk lattice constant of $5.43 \AA$.
The local-density approximation is used to describe electronic exchange and correlation. First, the total energy is minimized and the atomic coordinates of the structure are optimized. Second, the Kohn-Sham band structure and the corresponding wave functions are calculated from the selfconsistent Kohn-Sham potential.

The master equation, Eq. (2), is numerically integrated for conduction bands in an energy range of $0.6 \mathrm{eV}$ above the conduction band minimum. In order to check the convergence with respect to the Brillouin zone sampling, we compared k-point sets ranging from $5 \times 5 \times 1$ to $33 \times 33 \times 1 \mathbf{k}$ points. For a given number of layers, the relaxation time is converged for a mesh of $17 \times 17 \times 1$ and higher. Since the electron-phonon coupling enters only in a cumulative way into $\Gamma_{n \mathbf{k}}^{\text {in }}$ and $\Gamma_{n \mathbf{k}}^{\text {out }}$, the results are less sensitive to materialspecific details of the phonons than they are to the electronic states. At present, the electron-phonon coupling parameters from the bulk are used to couple to both electronic bulk and surface bands. For the surface bands, this may underestimate the electron-phonon coupling: Since the buckling of the $\mathrm{Si}$ surface dimers is sensitive to the occupation of the surface bands, a strong polaronic coupling is to be expected at the surface. However, the dynamical consequences of selective coupling mechanisms between electronic surface states and surface phonons are beyond the scope of our present study. The bulk phonon modes are introduced in terms of a simplified phonon model that allows for a transparent interpretation of the results. We approximate the full phonon spectrum $\omega_{i q}$ of the Si slab (see, e.g., Refs. 31 and 32) by using an effective two-mode model with one optical mode independent of the wave vector $\left(\omega_{1}=57 \mathrm{meV}\right)$ and one acoustical mode with linear dispersion in $\mathbf{q}\left(\omega_{2} /|\mathbf{q}|=6.1 \mathrm{meV} \mathrm{nm}\right)$. The matrix elements $D_{n \mathbf{k}, n^{\prime} \mathbf{k}^{\prime}, i \mathbf{q}}$ are proportional to the wave function overlap $\left\langle\varphi_{n \mathbf{k}}\left|e^{i \mathbf{q r} \mid}\right| \varphi_{n^{\prime} \mathbf{k}^{\prime}}\right\rangle$, with a factor $D_{i \mathbf{q}}^{\mathrm{def}}$ assumed to be constant (independent of $\mathbf{q}$ ) for optical phonons, while it scales like $\sqrt{\mathbf{q}}$ for acoustical phonons. ${ }^{17}$ Numerical values of $7.37 \mathrm{eV} \mathrm{nm}^{1 / 2}$ for the acoustical and $40 \mathrm{eV}$ for the optical mode have been adopted from literature. ${ }^{33,34}$

We now address the specific situation of the experiments of Ref. 6: The $\mathrm{Si}(100)$ surface is excited by an optical pulse with a photon energy $h \nu=1.69 \mathrm{eV}$ and a broadening of 40 meV, which corresponds to $t_{\text {pulse }} \sim 50$ fs. The pulse is incident perpendicular to the surface, with linear polarization diagonally oriented to the crystal reference frame with equal $x$ and $y$ components. The temperature is $T=90 \mathrm{~K}$, as defined by the phonon bath.

One characteristic feature of the $\operatorname{Si}(100)(2 \times 1)$ surface is an unoccupied surface band that is lower in energy than the bulklike conduction bands [upper bands in Fig. 1(a)]. For the time scales of interest here, it is this surface band where all excited electrons will end up after relaxation from energetically higher states (prior to exciton formation that takes place on a longer time scale ${ }^{6}$ ). The initial electron population $f_{n \mathbf{k}}$ after excitation by the pulse is plotted as a function of electron energy in Fig. 1(b). The population is determined by the relative strength of the allowed interband transitions and the pulse spectrum. It covers an energy range from 0 to about $0.55 \mathrm{eV}$, with a strong peak at $0.47 \mathrm{eV}$ (relative to the surface band minimum). This peak is due to the strong transition from the valence band top to the surface conduction band. 


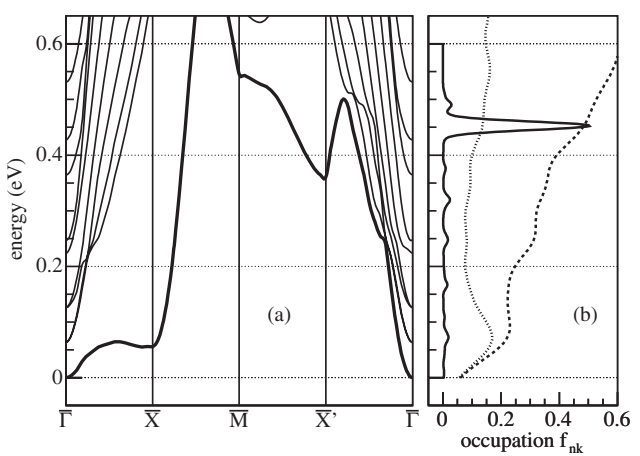

FIG. 1. (a) Band structure of a 30 layer $\mathrm{Si}(100)$ slab $\left(\bar{X}=(\sqrt{2} \pi / a, 0), \bar{M}=(\sqrt{2} \pi / a, \sqrt{2} \pi / a), \bar{X}^{\prime}=(0, \sqrt{2} \pi / a)\right.$. The lowest band is the surface band (thick); the higher bands are bulklike. (b) The initial occupation (solid), the total density of states (dashed), and the partial density of states for the surface band (dotted) are depicted. The density of states is smeared with a Gaussian function of width $0.05 \mathrm{eV}$. The zero of the energy scale is set the surface band state at $\bar{\Gamma}$.

The temporal relaxation dynamics after optical excitation is displayed in Fig. 2 (logarithmic scale). Here, the electron populations at $0 \mathrm{fs}, 100 \mathrm{fs}, 2 \mathrm{ps}$, and $190 \mathrm{ps}$ are shown. At $100 \mathrm{fs}$, the population at the band bottom $(0-0.2 \mathrm{eV})$ can already be found, while there remains a significant population in the higher bands. At $2 \mathrm{ps}$, an occupation peak is observable at $0.24 \mathrm{eV}$. This behavior suggests the existence of a phonon relaxation bottleneck near the minimum of the bulk conduction bands (around $0.2 \mathrm{eV}$ ). Due to this bottleneck, electron transfer and relaxation phenomena occur on two different time scales: (i) Efficient scattering of the electrons initially excited into the surface band by optical phonons leads to the fast initial increase in population at the surface band minimum. Simultaneously, electrons initially optically excited into bulklike states relax within the bulk band continuum. (ii) Multiple scattering processes between bulklike bands and finally into the (lower part of the) surface band leads to a delayed, slowly saturating population of the surface band minimum. Both time scales can be clearly observed in Fig. 3, wherein the population of the minimum energy state (located at the $\bar{\Gamma}$ point in the surface band) is plotted. The extracted values for the two time scales are 1 and $40 \mathrm{ps}$, respectively. The analysis shows that the relax-

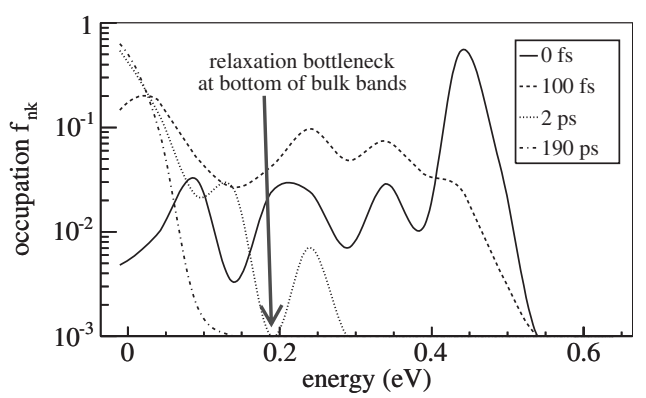

FIG. 2. Population as a function of energy for $0,100 \mathrm{fs}, 2 \mathrm{ps}$, and $190 \mathrm{ps}$ after optical excitation. At $t=2 \mathrm{ps}$, it is observed that part of the population still remains in bulklike electronic states above $0.2 \mathrm{eV}$ (relaxation bottleneck).

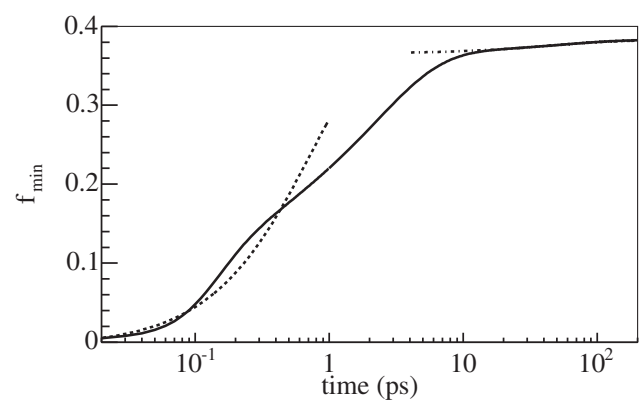

FIG. 3. Temporal evolution of the population of the surface band minimum (solid line) and exponential fits assuming two time scales for relaxation (dotted lines). For $t<0.5 \mathrm{ps}$, the population increase is governed by the fast time scale. The population saturates after $t=190 \mathrm{ps}$.

ation via bulklike optical phonons used in this study is much faster in comparison to that via acoustical phonons: the deformation potential for the optical phonons is larger; also, the energy dissipated by each optical phonon is higher. The scattering within the bulklike bands is dominated by optical phonons. The scattering from the bulklike states at the conduction band minimum to the surface state, however, can only be achieved by acoustical phonons. This is because the electrons that have relaxed to the minimum of the bulk conduction bands near $\bar{\Gamma}$ [second lowest band in Fig. 1(a)] are energetically very close to the surface band minimum [lowest band in Fig. 1(a)], and the energy difference between these two (initial and final) states is too small to allow for the emission of an optical phonon. This feature requires the surface to be modeled by a sufficiently thick slab for its correct description. ${ }^{35}$

The specific relaxation process involving two timescales and its explanation through electron-phonon processes is in agreement with the experimental results in Ref. 6. The short timescale found in the calculations agrees well with the findings ( $\approx 1$ ps for our calculation versus $1.5 \mathrm{ps}$ in experiment). The calculated timescale for the slower process, however, is still by a factor of 5 too fast compared to the experiment. Since it involves scattering of electrons from the bulk into surface states, it would probably require systems with an even larger bulk region to accurately model it.

In conclusion, we propose a linkage of the DMT and the DFT as a powerful method to investigate the electron dynamics at surfaces. In line with experimental findings, ${ }^{6}$ our calculations for the $\operatorname{Si}(100)(2 \times 1)$ surface show (i) a fast electron equilibration in the surface band on (picosecond time scale) due to efficient electron-optical-phonon scattering and (ii) a phonon bottleneck between bulklike and surface bands due to less efficient acoustical phonon scattering. The method enables us to identify the relaxation mechanisms specific to a particular surface structure.

We thank M. Weinelt, F. Willig, L. Töben, and L. Gundlach for fruitful discussions. This work was supported by the DFG (Research Centers SPP 1093, SFB 616, and CoE UniCat). 
*knorr@itp.physik.tu-berlin.de

${ }^{1}$ R. Haight, Surf. Sci. Rep. 21, 275 (1995); M. Baeumler and R. Haight, Phys. Rev. Lett. 67, 1153 (1991).

${ }^{2}$ G. D. Mahan, in Photoemission and Absorption Spectroscopy of Solids and Interfaces with Synchotron Radiation, edited by M. Campagna and R. Rosei (North-Holland, Amsterdam, 1990), pp. 25-40.

${ }^{3}$ H. Petek and S. Ogawa, Prog. Surf. Sci. 59, 239 (1998).

${ }^{4}$ L. Töben, L. Gundlach, T. Hannappel, R. Ernstorfer, R. Eichberger, and F. Willig, Appl. Phys. A: Mater. Sci. Process. 78, 239 (2004).

${ }^{5}$ S. Tanaka and K. Tanimura, Surf. Sci. Lett. 529, L251 (2003).

${ }^{6}$ M. Weinelt, M. Kutschera, T. Fauster, and M. Rohlfing, Phys. Rev. Lett. 92, 126801 (2004).

${ }^{7}$ S. Ramakrishna, F. Willig, and A. Knorr, Appl. Phys. A: Mater. Sci. Process. 78, 247 (2004).

${ }^{8}$ A. Zeiser, N. Bücking, J. Götte, J. Förstner, P. Hahn, W. G. Schmidt, and A. Knorr, Phys. Status Solidi B 241, R60 (2004); A. Zeiser, N. Bücking, J. Förstner, and A. Knorr, Phys. Rev. B 71, 245309 (2005).

${ }^{9}$ M. Reichelt, T. Meier, S. W. Koch, and M. Rohlfing, Phys. Rev. B 68, 045330 (2003).

${ }^{10}$ M. Lindberg and S. W. Koch, Phys. Rev. B 38, 3342 (1988).

${ }^{11}$ V. Axt and S. Mukamel, Rev. Mod. Phys. 70, 145 (1998).

${ }^{12}$ K. Hannewald, S. Glutsch, and F. Bechstedt, Phys. Rev. Lett. 86, 2451 (2001).

${ }^{13}$ I. Waldmüller, J. Förstner, and A. Knorr, Nonequilibrium Physics at Short Time Scales (Springer, Berlin, 2003).

${ }^{14}$ W. Hoyer, M. Kira, and S. W. Koch, Phys. Rev. B 67, 155113 (2003).

${ }^{15}$ I. Waldmüller, J. Förstner, S.-C. Lee, A. Knorr, M. Woerner, K. Reimann, R. A. Kaindl, T. Elsaesser, R. Hey, and K. H. Ploog, Phys. Rev. B 69, 205307 (2004).

${ }^{16}$ T. Feldtmann, L. Schneebeli, M. Kira, and S. W. Koch, Phys. Rev. B 73, 155319 (2006).

${ }^{17} \mathrm{H}$. Haug and S. W. Koch, Quantum Theory of the Optical and Electronic Properties of Semiconductors, 3rd ed. (World Scientific, Singapore, 1990).
${ }^{18}$ J. Li and D. A. Drabold, Phys. Rev. B 68, 033103 (2003).

${ }^{19}$ P. Rinke, A. Qteish, J. Neugebauer, C. Freysoldt, and M. Scheffler, New J. Phys. 7, 126 (2005).

${ }^{20}$ A. Knorr, S. Hughes, T. Stroucken, and S. W. Koch, Chem. Phys. 210, 27 (1996).

${ }^{21}$ S. Albrecht, L. Reining, R. Del Sole, and G. Onida, Phys. Rev. Lett. 80, 4510 (1998).

${ }^{22}$ A. Leitenstorfer, C. Fürst, A. Laubereau, W. Kaiser, G. Tränkle, and G. Weimann, Phys. Rev. Lett. 76, 3662 (1996).

${ }^{23}$ J. Schilp, T. Kuhn, and G. Mahler, Phys. Rev. B 50, 5435 (1994).

${ }^{24}$ F. Steininger, A. Knorr, T. Stroucken, P. Thomas, and S. W. Koch, Phys. Rev. Lett. 77, 550 (1996); D. Reiter, M. Glanemann, V. M. Axt, and T. Kuhn, Phys. Status Solidi B 243, 2297 (2006).

${ }^{25}$ M. Bonn, S. Funk, C. Hess, D. Denzler, C. Stampfl, M. Scheffler, M. Wolf, and G. Ertl, Science 285, 1042 (1999).

${ }^{26}$ L. Töben, L. Gundlach, R. Ernstorfer, R. Eichberger, T. Hannappel, F. Willig, A. Zeiser, J. Förstner, A. Knorr, P. H. Hahn, and W. G. Schmidt, Phys. Rev. Lett. 94, 067601 (2005).

${ }^{27}$ N. Buecking, P. Kratzer, M. Scheffler, and A. Knorr, Appl. Phys. A: Mater. Sci. Process. 88, 505 (2007).

${ }^{28}$ M. Rohlfing and S. G. Louie, Phys. Rev. Lett. 83, 856 (1999).

${ }^{29}$ P. Eggert, Ph.D. thesis, FU Berlin, 2005.

${ }^{30}$ J. Dabrowski and M. Scheffler, Appl. Surf. Sci. 56-58, 15 (1992).

${ }^{31}$ G. Nilsson and G. Nelin, Phys. Rev. B 6, 3777 (1972).

${ }^{32}$ P. Giannozzi, S. de Gironcoli, P. Pavone, and S. Baroni, Phys. Rev. B 43, 7231 (1991).

${ }^{33}$ J. C. Merle, M. Capizzi, P. Fiorini, and A. Frova, Phys. Rev. B 17, 4821 (1978).

${ }^{34}$ A. Blacha, H. Presting, and M. Cardona, Phys. Status Solidi B 126, 11 (1984).

${ }^{35}$ Considering the electronic band structure of thin slabs, transitions by optical phonons are allowed due to an overestimated energy gap between unoccupied surface and bulk states leading to a too fast relaxation dynamics. 